\title{
LÉGISLATION
}

\section{L'Installation des Lignes électriques sur les Propriétés privées (Déclaration de l'utilité publique et de l'urgence)}

\author{
Par Paul BOUGAUlt, Avocat à la Cour d'Appel de Lyon.
}

\begin{abstract}
La plupart des grands réseaux de distribution ou de transport de courant clectrique obtiennenl aujour.

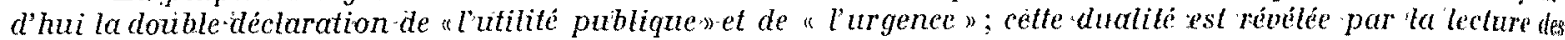
premières lignes de tout décret dans lesquelles l'an trouve ces mentions : "uu la loi du 15 juin 1906 " et un pen plus loin «vu la loi du 12 août 1919 ". Si la première de ces lois est à peu près connue des ćlectriciens, il n'en est pas de même de la seconde. De plus, pour ceux qui ignorent, mais,veulent ćtudier, il suffit de lire l'article 12 de la loi du 15 juin 1906 pour y trouver un ensenible de renseignements très facilenent complétés par l'article 38 du décret du 3 avril 1908 (pour les distributions) et les articles 19 et 20 du décret dul 24 avril 1923 (pour les lignes de transport-jonction). Au contraire, la loi sur l'urgence du 12 août 1919 nous renvoie seulement ì l'article 76 de la loi du 3 mai 1841 sur l'expropriation d'utilitć publique; et cel article 76 nous renvoie à la loi : du 30 mars 1831 relative aux travaux des fortifications.

A ussi, croyons-nous devoir nous contenter de résumer les principes fort connus de la loi du 15 juin 1906, pour accorder un développement un peu plus complet au texte de 1919 , étant donné surlout que quand on prend en lui-même, il ne signifie presque rien.
\end{abstract}

I

La Loi du 15 Juin 1906 et la Déclaration d'Utilité ptblique

Grâce à une procédure spéciale dont les grandes lignes ont été inspirees au rédacteur de l'article 12 de la loi du 15 juin 1906 par la łoi du 28 juillet 1885 sur. l'établissement des lignes télégrap̈niques et téléphoniques, tout réseã électrique bénéficiant de la déclaration d'utilité puiblique, peut constituer certaines servitudes très nettement déterminées qui ne comportent pas l'expropriation parce qu'elles n'affectent point la proprièté, mais efflenrent seulement la possession.

L'entrepreneur est donc autorisé à suivre le tracé approuvé par I'Administration ; et sur le parcours de ce tracé, il peut, pour des courants non dangereux pour le voisinage des maisons, ancrer des supports dans des façades, ou passer au-dessus des proprítés privées ; et, pour toutes sortes de courant, il peut placer sur des terrains non bâtis mi clos de murs, des canalisations aériennes avec leurs supports, ou des canalisations souterraines et ébrancher les arbres qui, se trouvant au contact des conducteurs aériens d'électricité, pourraient, par leur mouvement ou leur chute, occasionner des courts-circuits ou des avaries aux ouvrages.

L'indemnité qui est due est fixée ensuite par le juge de paix, car la loi ne prononce nulle part le mot d'indemnité préalable ; rien n'empêche qu'elle soit fixée à l'amiable ret qu'elle soit versée avant le passage des fils si la solution amiable ou judiciaire est intervenue auparavant. Ces questions d'alleurs ont déjà été éclairées par des décisions de justice (voir notamment l'arrêt de la Chambre Civile de la. Cour de Cassation en date du 12 juitlet 1921 , affaire Lissaraque contre Giros et $\mathrm{C}^{\mathrm{le}}$ et Ader, Dalloz 1921, 1.186).

Pour avoir un tracé approuvé par l'Administration, et s'en servir vis-à-vis des tiers, le distributeur doit se soumettre aux enquêtes prescrites par l'article 36 du décret du 3 avril 1908 dite enquête sur le parcellaire, laquelle comporte un Commissaire enquêteur nommé par le Préfet; si sur les observations faites à l'enquête, le tracé est modifié par le concessionnaire, ou bien s'il est maintenu, il est dans les deux cas envoyè par'T'Ingénieur en chef au Préfet qui le rend définitif par son approbation notifiée au concessionnaire. Pour les réseaux de transport-jonc. tion, l'article 36 du décret du 3 avril 1908 est remplacé par deux articles du décret du 24 avril 1923 qui stipulent comme différence que le pouvoir d'approbation du tracé appartient au Ministro au cas où il y aurait eu discussion à l'enquète (art. 19 et 20),

II

\section{La Procédure d'Expropriation en Cas d'Urgenge déclarét}

Mais, si les servitudes spéciales ne se rencontrent poim, c'est-à-dire s'il y a lieu notamment de grever de servitudes do passages aériens on souterrains, des terrains bâtis ou clos de murs, personne n'ignore qu'il faut recourir à la loi normale de l'expropriation : on isait encore que squand il $y$ a urgente déclarée, ce n'est point la loi du 3 mai 1841 qui est applicable, mais... une autre loi. On croit la trouver dans la loi du 12 aout 1919, qui est relative aux travaux urgents après la gucre (voir Lois Nouvelles, page 835); mais on n'y lit qu'un texte extrêmement court qui est ainsi conçu : "Article pro mier. - Pendant un délai de 5 ans à partir de la cessátion des hostilités, l'application de l'article 76 de la loi du 3 mai 1841 est étendue à tous travaux publics urgents... Art. 2. - La déclar. tion d'urgenae a aura lieu par décret."

Ce texte contient donc uniquement le principe que, lorsqut la déclaration d'urgence aura été obtenue par un décret, la pro* cédure d'expropriation aura lieu, d'après l'ant. 76 de la loi w 3 mai 1841.

C'est donc à ce dernier article que l'on se réfière; mais - nolt velle surprise - cet article, dans son premier paragraphe, ret. voie lui-même à une autre loi, celle du 30 mars 1831. "L'exptot priation ou T'occupation temporaire, dit ce paragraphe, en ch d'urgence, des propriétés privées qui seront jugées néccssaires pour des travaux de fortification continueront d'avoir lisu conformément aux dispositions prescrites par la loi du 30 mafs 1831."

Il se dégage donc, à première vue, des lignes qui précèdent que, de renvoi en renvoi, l'Ingénieur est autorisé à croire qu'nyant abouti à ce texte du 30 mars 1831, il sera dispensé pour toujouls 
Je s'vccuper de la loi du. 3 mai 1841 ; malheureusement, le: deuxiène paragraphe de l'art. 76 contient quelques mots qui paraissent impliquer une certaine réserve: "Toutefois, est-il dit dans le deuxième paragraphe, lorsque les propriétaires ou autres intéressés n'auront pas accepté les offres de l'Administration, le règlement délinitif des indemnilés aura lieu conformément aux dispositions du titre. IV ci-dessus. "

Arrives à ce point, nous avons entendu les fonctiomaires les plus élevés de l'Administration déclarer qu'ils se refusaient à comprendre... quoi que ce soit.

Voldi la réalité : Lous ces textes sont très facilement intelligibles ou, au contraire, absolument obscurs, selon que le lecteur connaît ou ne connaît pas l'origine des lois relatives à l'expropriation, et l'on aurait tort de croire que cet historique est très compliqué : il suffira d'en lire le résumé dans les lignes qui suivc1!!.

\section{III}

Diverses Etapes de la Législation. -- Principes actuels et Pringipes anciexs

On a aujourd hui une Lelle habitude d'envisager l'expropriation tolle qu'elle est établie par la loi organique du 3 mai. 1841, que les prineipes actuels nous paraissent avoir toujours existé. Le poumoir excutif, déclare, en principe, l'utilité publique ; le pouvoir judiciuire prononce l'expropriation, c'est-à-dire la rupture du lien de propriété ; rautorité préfectorale désigne les parcelles frappies; le Jury d'expropriation fixe souverainement les indemnitis.

Or, ces grandes règles qui nous paraissent presque banales à force d'ètre appliquées quotidiennement, ont été longues à s'implanter dans notre droit.

Sans doute, le Code Civil dans son art. 545 avait bien déclaré inviolable la propriété, si ce n'est pour cause d'utilité publique, et moyennant une juste et préalable indemnité ; mais il n'avait attribué à aucun service spécial, la compétence voulue soit pour déclarer l'utilité publique, soit pour fixer l'indemnité.

Il en résulta cue, d'après un avis du Conseil d'Etat du 18 août 1807 , le pouvoir administratif prit pour lui tous les droits, en assumant tontes les charges : c'est-à-dire à la fois la déclaration, l'expropriation, le règlement de l'indemnité. Peu de fonctionnaires se doutent, quand ils s'occupent de dessèchement des marais en conformité de la loi du 16 septembre 1807 , que ce texte n'est que l'application actuelle de ces très anciens principes. Cela démontre qu'aujourd'hui encore des lois de très vieille date ont conservé toute leur vigueur.

Ce fut la loi du 8 mars 1810 - date que l'on voudra bien retcnir - qui fit, la première, une séparation très nette entre les pouvoirs de l'administration et ceux de l'autorité judiciaire ; à l'administration, ćchut toute compétence pour déclarer l'utilité publique, el préparer la prise de possession; à l'autorité judiciaire (c'est-ì-dire au Tribunal Civil) fut confié le soin de prononcer l'exproprialion -... en fixant l'indemnité - : le Jury d'expropriation ne fut pas encore institué, à ce moment.

Nêrnmoins; la loi du 8 mars 1810 était, sur certains points, très complète : In déclaration d'utilité publicue devait ètre faite par bécrel impérial ; la prise de possession devait ètre précédée d'uncenquète et d'un arrêté préfectoral désignant les propriétés: particulières frappées d'expropriation. C'est l'apparition véritable de l'arrêté que nous appelons aujourd"hui "l'arrêté de cessibilite'" ; il avait alors pour but now' seulement de permettre aux particuliers de faire valoir leurs observations ; mais aussi, et c'ćlait le désin évident di légishateur, - de voir les proprié- taires bien renseignés sur les emprises futures, entrer en tractation avec l'Administration pour établir d'un commun accord le montant de l'indemnité:

Si cet accord n'intervenait pas, il appartenait au Tribunal, sur la demande du Préfet, d'envoyer l'Administration en possession provisoire ; c'était aux propriétaires à faire opposition devant le Tribunal, dans le cas où ils auraient estimé que des irrégularités de procédure dans les enquêtes s'étaient produites ; le Tribunal pouvait alors, soit donner raison aux particuliers et enjoindre à l'Administration de surseoir jusqu'à régularisation complète, soit rejeter leur demande; dans ce dernier cas, il procédait lui-même à la fixation de l'indemnité en s'aidant au besoin de renseignements demandés par lui à des experts.

Dans tous les cas, le Tribunal avait le droit absolu qui lui était conféré par l'article 19 de la loi, d'autoriser, pourvu qu'il y ent urgence, la mise en possession rapide de l'Administration.

Cette loi du 8 mars 1810 était en vigueur (elle formait donc le droit commun en matière d'expropriation) quand la loi du 30 mars 1831 -.- celle que nous devons appliquer aujourd'hui fit son apparition. Elle a été créée pour prévoir dés travaux spéciaux ; car elle porte comme titre "loi relative à l'expropriation temporaire, en cas d'urgence, des propriétés privées nécessaires aux tranaux de fortification".

La.loi du 30 mars 1831 a pour but de permettre une rapidité plus grande, par rapport à la loi du 8 mars 1810; on n'en saurait douter lorsqu'on en lit le premier article : " lorsqu'il y aura lieu d'occuper tout ou partie d'une ou de plusieurs propriétés particulières pour $y$ faire des travaux de fortifications dont l'urgence ne permettra pas d'accomplir les fórmalités de la loi du 8 mars 1810; il sera procédé de la manière suivante :..... *

Sur quelle partie de la loi du 8 mars 1810 , l'augmentation de rapidité indiquée par la loi du 30 mars 1831, fait-elle sentir son influence ? La lecture des onze premiers articles de la loi suffit pour nous renseigner et pour nous permettre de répondre que cet accroissement de rapidité se trouve danswa suppression des formalités dites "préfectorales" ordonnées par la loi du 8 mars 1810. Alors que, comme il a été exposé ci-dessus, la loi du 8 mars 1810 impose, après l'apparition du décret, une procédure par laquelle le Préfet, après enquête, prend un arrêté désignant les parcelles auxquelles l'expropriation est applicable, nous- voyons la loi du 30 mars 1831 poser les règles suivantes : le Préfet dans les 24 heures de la réception du décret, en transmet ampliation au Procureur de la République, qui saisit le Tribunal Civil, lequel rend un jugement ordonnant que l'un des juges se transportera sur les lieux avec un expert que le Tribunal nommera d'office (art. 3 de la loi du 30 mars 1831).

Le juge commis rend une ordonnance pour fixer le jour et l'heure de sa descente sur les lieux, et le Procureur de la République signifie cette ordonnance au Mairc de la commune et à l'expert nommé par le Tribunal. Le Waire convoque tous les propriëtaires intéressés qui lui sont désignés par l'agent chargé de la direction des travaux, et les personnes ainsi convoquées peuvent se faire assister également par un expert. Mais ce n'est pour elles qu'une pure facilité (art. 4- de la loi du 30 mars 1831).

La partie la plus essentielle des formalités, celle qui supprime le plus radicalement les longueurs qui auraient résulté de l'enquête sur le parcellaire et de l'arrèté préfectoral aujourd hui supprimés se trouve dans:les articles $5,6,7$ et 8 de la loi de 1831 . D’après lesdits articles, au jour fixé par le juge-commissair, doivent se trouver sur les lieux : un agent de l'Administration des. domaines et un Ingénieur, désignés tous: les deux par le Préfet: ils y rencontrent le Whire, l'Ingénieur chargé des travaux et l'expert désigné par le. Tribunal. Le juge-commissaire reçoit 
le serment des experts, en fait mention sur le procès-verbal, et, sur le terrain, l’Ingénieur dirigeant les travaux qui remplace évidemment l'agent militaire indiqué par la loi, détermine en présence de tous, par des pieux et piquets le périmètre du terrain dont l'exécution des travaux nécessite l'occupation. On peut dire que ce piquetage remplace l'enquète et l'arrèté préfectoral, puisqu'il est un acte matériel accompli par l'agent de l'Administration sous les yeux des parties, ou au moins après la convocation régulière de celle-ci, et que l'emprise est vraiment effectuée.

Cette opération étant achevée, chacun des experts qui se trouvent sur les lieux, aura à effectuer sa mission : à celui qui a été désigné par le Préfet, il incombe, de concert avec l'agent de l'Administration, de procéder à la levée du plan parcellaire, pour indiquer dans le plan général de circonscription, les limites et la superficie des propriétés particulières. (1).

Quant à l'expert nommé par le Tribunal il dresse un procèsverbal qui comprend la désignation des lieux, cultures, plantations, bâtiments, clôtures et autres accessoires des fonds. Cette désignation doit être assez complète pour permettre ultérieurement la fixation des dommages pour changements ou dégâts. Et il estime également la valeur de chaque parcelle, ainsi que l'indemnité qui pourrait ètre due pour tous dommages. Cette partie du rapport de l'expert nommé par le Tribunal, est faite contradictoirement avec l'agent de l'Administration et l'expert nommé par le Préfet, et bien entendu avec les parties intéressées.

La deuxième partie de la fonction de l'expert nommé par le Tribunal pourrait être appelée la rédaction d'un résumé ; il l'accomplit seul, puisqu'il lui suffit, en indiquant la nature de chaque propriété, de donner les motifs des évaluations diverses, de transcrire l'avis de chacune des parties présentes, et de faire signer les dires qu'il aura consignés par les personnes qui les auront formulés (article 8 de la loi du 30 mars 1831).

C'est ainsi que nous trouvons dans ledit article 8 la clôture de cet ensemble de constatations à la fois judiciaires et administratives, voulu par le législateur dans le but de déterminer les parcelles à occuper, en décrire l'état, permettre aux parties de se rendre compte de l'ouvrage projeté.

Nous ne serons donc point étonnés de rencontrer maintenant l'article 9, qui va prévoir l'accord de l'Administration et des parties en ces termes : "Lorsque les propriétaires ayant le libre

(1) Est-il besoin de dire que la loi n'a jamais voulu obliger l'expert nommé par le Préfet à lever immédiatement le plan parcellaire. Comme le fait très bien remarquer Dalloz (Code des Lois Politiques et Administratives, annoté, Vo Travaux Publics No 6033 et suivants) pour éviter toute perte de temps, le périmètre des terrains peut être tracé à l'avance, ainsi que le plan parcellaire, de manière qu'il ne reste qu'à vérifier les points dont l'exactitude serait mise en doute ; cela résulte au surplus de la discussion de la loi du 30 mars 1831. L'expert du Préfet peut même, sans dresser procès-verbal du plan parcellaire, se borner à signer le plan déjà tracé en y joignant un tableau indicatil des noms des propriétaires, tels qu'ils sont indiqués sur la matrice des rôles et la contenance de chaque parcelle. Et comme le plan est toujours dressé à l'avance, il suffit que le juge-commissaire mentionne, dans son procès-verbal, que ce plan a été reconnu exact, ou que la vérification réclamée sur certains points, a donné tels ou tels résultats.

Il nous paraît tomber sous le sens que le désir du législateur est que, d'une part, les parties soient mises à même de bien se rendre compte du tracé qu'elles n'ont point à discuter puisqu'il est déterminé par l'entreprise, et que, d'autre part, il soit fait un plan pour conserver la trace très exacte de l'état des lieux ; ce dernier point n'a vraiment d'importance que pour les travaux de fortifications qui englobent et font disparaître quelquefois tout ce qui est sur une parcelle : il est évident que pour la simple occupation d'une ligne de transport dans un terrain clos de murs, les constatations ont une importance infiniment moindre. exercice de leurs droits, consentiront à la cession qui sera du. mandée et aux conditions qui leur seront offertes par l' $\Lambda$ dministration, il sera passé entre eux et le Préfet un acte de vente qui sera rédigé en la forme des actes d'administration et dont la minute restera déposée aux archives de la Préfecture. "C'est l'article qui sera toujours vrai : la possibilité d'un accord amiable relativement à l'indemnité est ardemment souhaitée par le législateur. Quand il intervient, il met fin aux formalités judiciaires, puisque c'est le Préfet qui tient la plume pour rédiger l'acte d'acceptation réciproque.

Mais, s'il ne peut y avoir lieu à contrat, par suite d'une difference trop considérable entre l'offre et la demande, la loi de 1831 ne veut pas que toute une opéralion soit suspendue pour une divergence de vues. L'article 10 déclare que le Tribunal sur le vu de la minute du procès-verbal dressé par l'expert judiciaire et de celui du juge-commissaire, détermine : l'indemnité de déménagement à payer aux délenteurs avant l'occupation, et une indemnité approximative de dépossession : cette seconde indemnité prend le nom de "provisionnelle ".

En payant la première, et en consignant la seconde, le Préfet acquiert le droit d'entrer immédiatement en possession, c'està-dire dès l'expiration d'un délai très court imparti aux particuliers pour évacuer les lieux.

L'article onzième déclare, dans son premier alinéa, - un peu inutilement peut-être - que cette indemnité provisionnelle ne portera aucun préjudice à la fixation de l'indemnité définitive; c'est le propre des "provisions » de ménager toujours le "délinitif».

Mais, nous ne saurions attacher trop d'importance à une remarque infiniment plus intéressante : les premières lignes de l'article 11 sont les dernières que nous ayons à lire dans la loi du 30 mars 1831 ; en d'autres termes, tout ce qui est contenu dans la fin de cet article et dans les articles suivants, nous est indifférent.

Car le moment est venu de nous rappeler la phrase qui dans l'article 76 de la loi du 3 mai 1841 (article par lequel nous avons été renvoyés à la loi du 30 mars 1831 ) commence par le mot "toutefois".

Et cette phrase est ainsi conçue : "Toutefois, lorsque les propriétaires ou autres intéressés n'auront pas accepté les offres de l'Administration, le règlement définitif des indemnités aura lieu conformément aux dispositions du titre IV de la présente loi \%.

Or, le titre IV de la loi du 3 mai 1841 c'est celui qui, pour le règlement des indemnités, prévoit la convocation des intéressés devant le jury, et la constitution de ce Tribunal très spécial connu sous le nom de jury.

C'est la grande hypothèse classique, le retour au droit commun trop connu pour que nous ayons à nous en préoccuper dans cette étude.

IV

\section{Résumé et Observations particulières}

Après avoir parcouru le cycle de tous ces principes, il nous est facile de répondre à cette question : quelle est la portée de la loi du 12 août 1919 ? Ce n'est pas elle qui a créé la procédure d'urgence : elle ne dit même pas que pendant les cinq années qui suivront la cessation des hostilités, tous les lravaux déclarés d'utilité publique bénéficieront d'une déclaration supplémentaire d'urgence : elle est beaucoup plus modeste... et beaucoup plus pratique : dans son article 2 (voir ci-dessus) cette petite loi si courte permet de faire déclarer d'urgence pendant lo délai 
précité, tous les travaux d'utilité publique par un décret. Si donc le decret était nuet sur celte déclaration, il n'y aurait point de procédure speciale. Mais si, au contraire, cette déclaration existe, la loi nous prévient que nous avons à appliquer la procédure de la loi du 30 mars 1831.

Etait-il ulile de le dire ? El quelle est la portée de cet appel à la loi de 1831 ?

11 nc faut pas oublier que, mêne au cas oủ la loi du 12 août 1919 n'aurail pas existé, nous aurions eu à appliquer par le seul fait que nous avions une déclaration d'urgence, une procédure spéciale. Mais, ce n'aurait point élé la procédure de la Ioi du 30 mars 1831.

On ne saurait oublier, en effet, que la loi du 3 mai 1861 prévoit que certaines expropriations - même complètement indépendanles de toute idée de fortification - demanderont à être menées très rapidement ; aussi, elle y consacre ses articles 65,66 , 67 el stuivants jusqu'à 74. Mais il sulfit de lire les articles précités pour remarquer que la procédure qui y est expliquée ne commence qu'après le jugement d'expropriation, c'est-à-dire qu'elle n'a aucune influence sur la partie administrative qui précède le jugoment et qu'elle n'intervient en rien à l'arrêté de cessibilité qui, dans la procédure ordinaire, est bien antérieur au jugement qui fait tomber, au profit de l'expropriant, l'inviolabilité de la propriété privéc.

Au contraire, si tout ce qui précède a été suffisamment clair, on a dû comprendre que la loi du 30 mars 1831 a pour but de supprimer l'enquete parcellaire ou plus exactement de la rendre plus matcriclle, plus tangible, puisqu'au lieu de se faire sur un plan, elle se fait par un piquetage en présence de deux experts, d'un juge-commissaire et des parties dûment appelées.

Si donc dans une procédure d'urgence, on ne suit pas la loi de 1831 , c'est-à-dire sí on oublie la loi du 12 août 1919 qui nous l'impose, il faudia suivre toutes les formalités écrites dans les articles 4 el suirants, jusqu'à 12 inclus, de la loi du 3 mai 1841 . Or, si l'urticle 12 diminue le poids et la longueur de ces formalités pour les expropriations poursuivies par une commune ou ayant trail aux travaux d'ouverture des chemins vicinaux elles conservent toule leur rigueur quand l'entreprise doit avoir lieu dans plusieurs départements, ou communes. D'après l'article 8 il doit y avoir une commission présidée par le Sous-Préfet ne pouvant délibérer qu'avec la présence de cinq membres, elle fonctionne pendant huit jours... etc...

On ne se trouve donc pas seulement en présence d'une différence de mots.

II. - En déclarant que l'article 76 de la loi de 1841 , c'est-à-dire pratiquement les onze premiers articles de la loi du 30 mars 1831 , serait applicable pendant cinq ans à partir do la cessation des hostilités, c'est-ì-dire jusqu'at mois d'octobre 1924 à tous les travaux declárés urgents (1), la loi du 12 août 1919 n'a pas voulu

(1) Le député Paisant, (Chambre des Députés, No 6015, annexe à la séance du 24 inai 1923) a déposé une proposition de loi tendant nous forcer à copier servilement les termes mêmes de la loi de 1831. Est-il besoin de dire que l'agent militaire sera remplacé par l'ingénicur de l'entreprise ? On peut même se demander si le Préfet convoquera l'agent des domaines dont la présence serait presque étrange, puisque son rôle, s'il se comprend très bien dans un travail de fortification s'incorporant au domaine de l'Etat, est nul dans les affaires de distribution. Il ne nous appartient pas d'ailleurs de le décider, puisqu'en tout cas, il ne gênera personne.

III. - Dans la loi de 1831, nous citons seulement pour mémoire et afin qu'il ne produise aucun trouble l'article 13 qui déclare : "l'occupation temporaire ne pourra avoir lieu que pour des propriétés non bâties. La simple remarque faite ci-dessus, d'après laquelle nous n'avons pas à connaître le texte de la loi du 30 mars 1831 dans la partie écrite à la suite du premier alinéa de l'article onzième suffirait pour nous rassurer ; de plus, personne ne confondra l'occupation temporaire avec l'expropriation, et c'est de l'expropriation que nous nous occupons en faisant remarquer qu'elle s'applique à tous les immeubles.

à proroger pour une nouvelle durée de cinq années les dispositions de la loi du 12 août 1919 : la Commission des Travaux Publies et des moyens de communication est saisie de cette proposition.

Il y est expliqué que l'article 76 de la loi du 3 mai 1841 "se "réfère aux dispositions de la loi du 30 mars 1831, aux termes des"quelles, d'une part; les travaux sont autorisés par un décret qui " en déclare en même temps l'urgence; d'autre part, l'enquête par" cellaire et l'arrêté de cessibilité du titre 2 de la loi de 1841 sont " remplacés par une simple expertise, d'après le procès-verbal de " laquelle le Tribunal fixe une indemnité de déménagement ou urẹ " indemnité provisionnelle et approximative de dépossession, les "détenteurs étant tenus d'abandonner les lieux dans les délais " qui ne peuvent excéder cing jours ou dix jours. "

" L'intérêt d'une telle procédure, dit-il, n’a pas à être clémontré, " le nombre des travaux que l'on peut qualifier d'urgents étant * forcément considérable au lendemain de la guerre. L'exposé " des motifs de la loi dn 12 août 1919 en cite quelques exemples : " on peut en signaler d'autres non moins caractéristiques, tels que " reconstruction d'ouvrages détruits avee des dispositions diffé" rentes, doublement des écluses de certains canaux, rectification " de courbes.

"Un certain nombre de ces travaux sont d'ores et déjà en " cours. Mais il en reste beaucoup d'autres à entreprendre, dont " l'urgence égale celle des premiers, leur importance ne le cédant " en rien pour la mise en valeur des ressources nationales et le " prompt relèvement économique du pays. Ceux-ci non plus ne " sauraient s'accommoder de la lenteur inhérente à la procédure " normale.

"Il est à remarquer d'ailleurs que le projet de loi présenté en " 1919 prévoyait la mise en application des nouvelles dispositions " pendant une période de dix ans, et ce n'est qu'au cours de la " discussion parlementaire, que ce délai a été réduit à cinq ans.

"Pour toutes ces raisons, il paraît indispensable de proroger " pour cinq nouvelles années l'effet de la loi du 12 aout 1919. Les " dispositions qu'elle contient présentent un intérêt de premier " ordre pour le pays, mais elles ne peuvent en aucun cas, léser les " propricetaires ou locataires en cause, le règlement définitif " des indeminités étant ajourné, lorsqu'il y a désaccord entre les " parties pour être effectué conformément au titre-IV de la loi de "1841. Ces dispositions n'offrent donc que des avantages." 